\title{
Divertículo rectal sintomático
}

\section{Symptomatic rectal diverticulum}

Álvaro Andrés Gómez V., MD. ${ }^{*}$

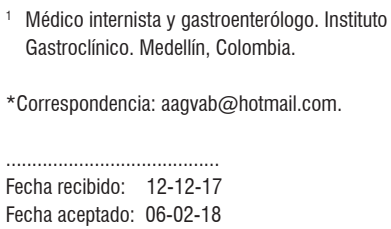

\begin{abstract}
Resumen
Los divertículos rectales son hallazgos inusuales durante la colonoscopia o estudios radiológicos del colon. Se estima una prevalencia aproximada del $1 \%$ y se presenta con mayor frecuencia en hombres entre la sexta y novena década de la vida. En la mayoría de pacientes son asintomáticos, aunque algunos pueden cursar con síntomas o complicaciones relacionadas. Se reporta el caso de una paciente con divertículo rectal grande con dolor abdominal y estreñimiento.
\end{abstract}

\section{Palabras clave}

Recto, divertículo, estreñimiento, dolor abdominal.

\begin{abstract}
\section{Keywords}

Rectum, diverticulum, constipation, abdominal pain.
\end{abstract}

Rectal diverticula are unusual findings during colonoscopy and radiological studies of the colon. Prevalence is estimated to be approximately $1 \%$, and they occur more frequently in men between the $6^{\text {th }}$ and $9^{\text {th }}$ decade of life. Most patients are asymptomatic, although some may present symptoms or related complications. We report the case of a patient with a large rectal diverticulum with abdominal pain and constipation.

\section{INTRODUCCIÓN}

La presencia de divertículos en el recto es un hallazgo infrecuente. En la mayoría de pacientes son asintomáticos y el diagnóstico suele ser incidental. Se pueden relacionar con diverticulosis colónica o como complicación de cirugías en el recto. Se presenta el caso de una paciente con divertículo rectal grande con dolor abdominopélvico y evacuación rectal incompleta.

\section{PRESENTACIÓN DEL CASO}

Mujer de 53 años sin antecedentes patológicos o quirúrgicos, quien desde hace 2 años estaba presentando dolor abdominal cólico recurrente de predominio en la fosa ilíaca izquierda que se irradiaba al hipogastrio y empeoraba con el consumo de alimentos, asociado con estreñimiento y deposiciones en la escala de Bristol entre 1 y 2. Destacó en el interrogatorio sensación de evacuación incompleta, tenesmo, con necesidad de pujo y mejoría marcada del dolor abdominal y pélvico posterior a la defecación. Negó sangrado rectal o pérdida de peso. El examen físico incluido el tacto rectal fue normal.

Por su edad y los síntomas referidos se decidió llevar a colonoscopia con el objetivo de descartar una patología orgánica estructural que explicara sus síntomas. En la pared lateral del recto medio a los $6 \mathrm{~cm}$ de la línea dentada, se observó un divertículo de unos $3 \mathrm{~cm}$ de diámetro, redondo, 
sin cambios inflamatorios ni lesiones en la mucosa que lo recubre, sin fecalitos y que cambia de forma con la insuflación/succión del aire (Figuras 1 y 2). Durante la exploración de los demás trayectos del colon se observaron múltiples divertículos con diámetros entre los 5 y $10 \mathrm{~mm}$ sin complicación (Figura 3). En el colon transverso, además, se resecó con pinza un pólipo diminuto de aspecto adenomatoso (Figura 4). Considerando que los síntomas de la paciente podrían explicarse por divertículos del colon (en especial aquellos sugestivos de trastorno en la evacuación por el gran divertículo rectal), se indicó el manejo con suplemento de fibra con Psyllium y antiespasmódicos, con lo que se presentó una mejoría importante del cuadro clínico y de su calidad de vida.

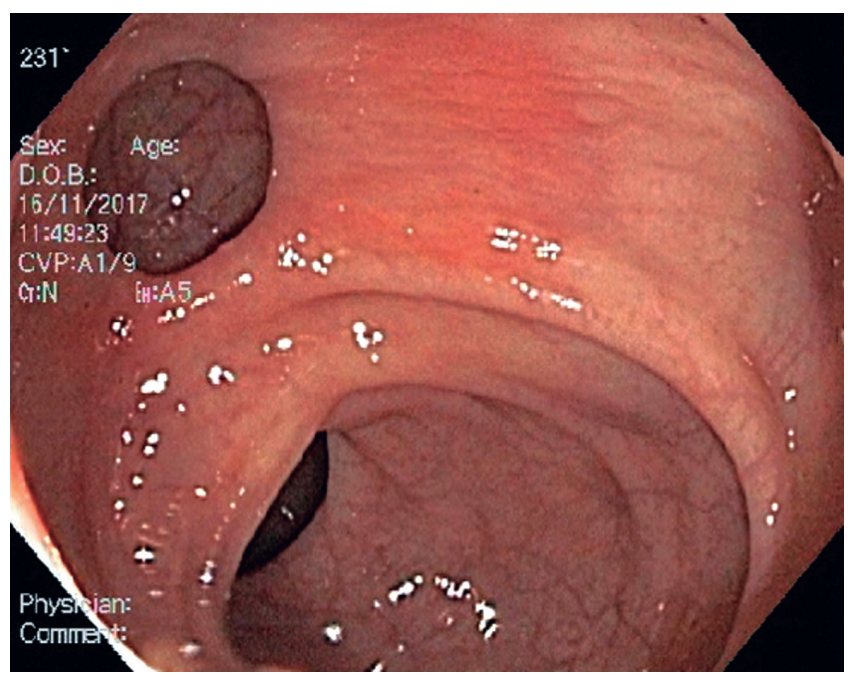

Figura 1. Divertículo rectal.

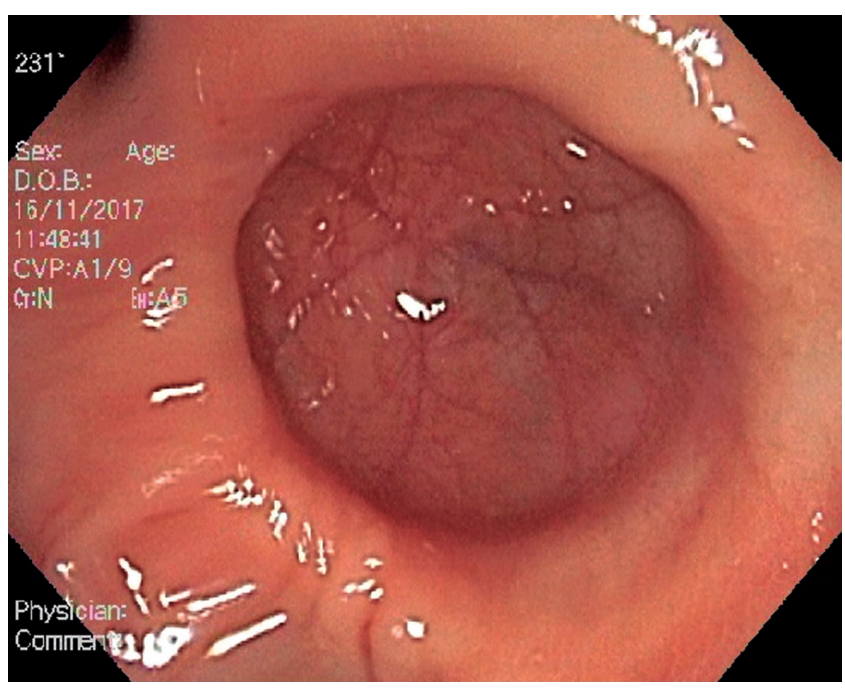

Figura 2. Mucosa al interior del divertículo rectal sin anormalidades.

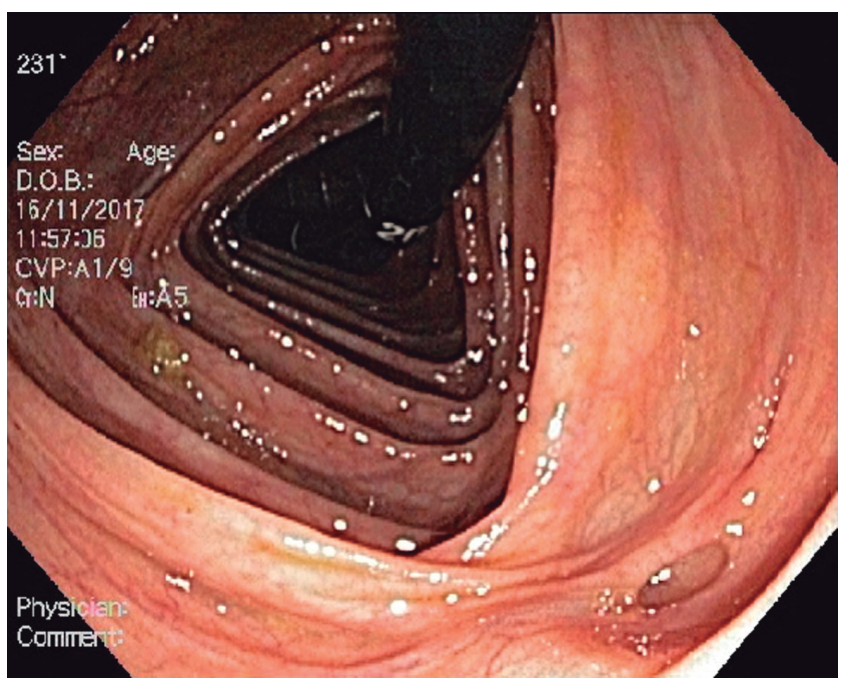

Figura 3. Retroflexión del colon derecho con divertículo.

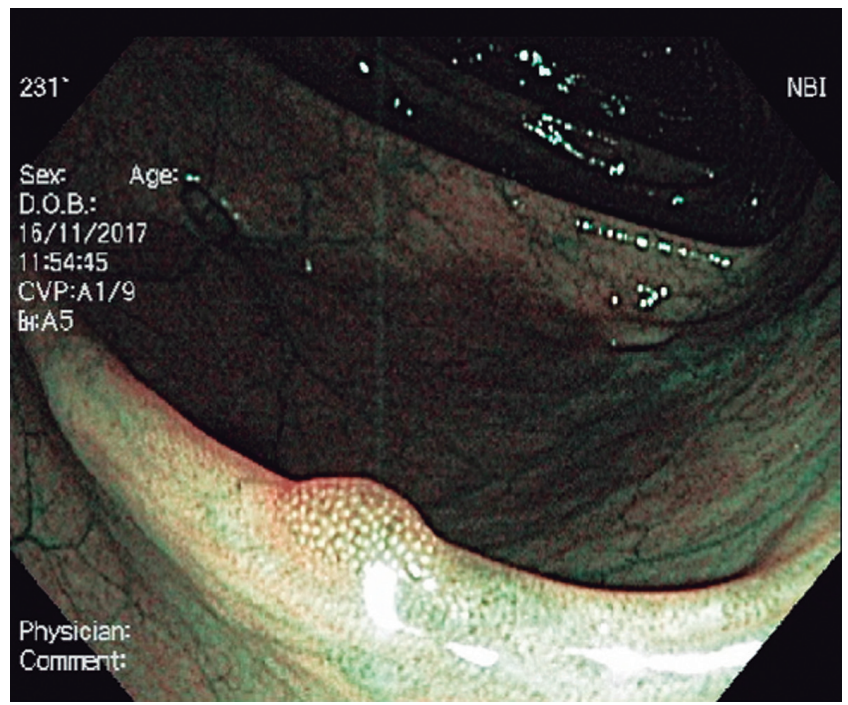

Figura 4. Pólipo adenomatoso de colon.

\section{DISCUSIÓN}

A diferencia de los divertículos en el colon, los divertículos en el recto contienen todas las capas de la pared, considerándose verdaderos divertículos (1). La verdadera prevalencia de los divertículos rectales es difícil de establecer dado que en la mayoría de los casos son asintomáticos y el diagnóstico suele ser incidental. Se estima que en la literatura se han publicado aproximadamente 80 casos. En Colombia solo se había reportado un caso en un paciente masculino como hallazgo incidental en colonoscopia (2). Una serie basada en estudios del colon por enema en 4854 pacientes encontró una prevalencia de $0,08 \%$ (3). Otro estudio más reciente (publicado en 2013) con una metodología similar 
encontró en 2877 pacientes una prevalencia del 1,5\% (4). Una serie de casos de pacientes llevados a colonoscopia encontró una prevalencia en 10 años del 0,15 \% y del 0,75 $\%$ en pacientes con diverticulosis colónica (5). En la mayoría de reportes, el compromiso es mayor en hombres entre los 55 y 85 años (6).

Tres teorías explican la baja prevalencia de este hallazgo. La disposición anatómica de las fibras musculares de la Tenia libera y la Tenia omentalis en el recto, en especial en las paredes anterior y posterior, llevan a reforzamiento de la estructura rectal y a mayor resistencia a variaciones intraluminales, lo cual a su vez explica por qué la mayoría de casos se ha documentado en la pared lateral (7). De igual manera, el recto presenta menor presión y menores movimientos peristálticos en comparación con el sigmoide, por lo cual la tasa de divertículos es mucho menor a la de este segmento colónico. Finalmente, dado el reporte de un caso de divertículo rectal en un paciente pediátrico, no se descarta que tenga un origen congénito (8).

Aunque la mayoría de casos se presenta con divertículos en los demás trayectos del colon, siendo este el principal factor de riesgo, un grupo de casos reportados tiene un origen iatrogénico posterior a cirugías como el procedimiento para hemorroides prolapsadas ( $\mathrm{PPH})$, anomucopexia circular de longo o la resección transanal rectal por prolapso mucoso (9-11). Otros factores de riesgo asociados incluyen obesidad, estreñimiento, episodios de impactación fecal recurrente, ausencia de soporte pélvico como el coxis, infecciones rectales, trauma o enfermedades musculares degenerativas (5). En la mayoría de casos, el diámetro de los divertículos rectales se presenta desde los $2 \mathrm{~cm}$ en adelante (12). Aunque en general no generan síntomas, algunos casos reportados incluyen todas aquellas complicaciones de los divertículos del colon (sangrado, diverticulitis y perforación). También se han descrito asociados a trastornos de la evacuación, con formación de fístulas, con ulceración sobre su superficie, asociados con prolapso rectal, con sensación de masa perineal e inclusive se ha descrito aparición de adenocarcinoma en el interior de un divertículo rectal $(13,14)$. Por esta última razón se recomienda hacer seguimiento periódico dado que al parecer podría haber un aumento en el riesgo de cáncer en su interior.

En el caso de la perforación, esta conlleva un menor grado de complicaciones en comparación con la que ocurre en un divertículo colónico, dada la ubicación anatómica del recto por debajo de la reflexión peritoneal (5). La documentación de los divertículos puede realizarse mediante colonoscopia o estudios radiológicos, desde colon por enema con bario o imágenes por tomografía axial computarizada (TAC) o resonancia magnética (RNM). En los estudios endoscópicos se debe tener precaución dado el riesgo de perforación
(15). En uno de los casos reportados se realizó manometría anorrectal convencional sin encontrar anormalidades en la presión o sensibilidad rectal, y tampoco en la presión del esfínter anal (16).

El manejo depende de la intensidad de síntomas y las complicaciones asociadas. Para pacientes con síntomas leves a moderados, se sugiere brindar una dieta rica en fibra y antiespasmódicos. Para casos con síntomas que alteran la calidad de vida del paciente y refractarios al manejo médico o con complicaciones relacionadas (sangrado, diverticulitis, perforación) se sugiere la cirugía. En este contexto se ha descrito desde la diverticulectomía hasta procedimientos de mayor complejidad como la rectosigmoidectomía o resección abdominoperineal $(17,18)$.

El caso de la paciente demuestra que pueden existir síntomas asociados con divertículos rectales grandes, en especial relacionados con limitación para una adecuada evacuación anorrectal. No se realizó manometría anorrectal debido a la mejoría con fibra, pero es una opción en pacientes refractarios dado que esta patología puede relacionarse con trastornos de la defecación, y podría tener algún rol en el tratamiento la terapia de retroalimentación o biofeedback.

\section{CONCLUSIONES}

Los divertículos rectales son un hallazgo infrecuente durante la colonoscopia o estudios radiológicos del colon. Deben sospecharse en pacientes con trastorno de la evacuación (como en el caso de la paciente) y en aquellos con antecedente de cirugía en el recto, o podría presentarse con complicaciones derivadas del divertículo como sangrado, diverticulitis o perforación. El manejo depende de la severidad de los síntomas y las complicaciones presentadas.

\section{REFERENCIAS}

1. Halpert RD, Crnkovich FM, Schreiber MH. Rectal diverticulosis: a case report and review of the literature. Gastrointest Radiol. 1989;14:274-6. doi: 10.1007/BF01889215.

2. Ángel A, Cardona D. Divertículo rectal. Reporte de un caso. Biosalud. 2017;16(2):119-22.

3. Walstad PM, Sahibzada AR. Diverticula of the rectum. Am J Surg. 1968;116:937-9. doi: 10.1016/0002-9610(68)90471-6.

4. Lohsiriwat V, Suthikeeree W. Pattern and distribution of colonic diverticulosis: Analysis of 2877 barium enemas in Thailand. World J Gastroenterol. 2013;19(46):8709. doi: 10.3748/wjg.v19.i46.8709.

5. Fagundes RB, Motta GL, Fontana K, Fonseca CB, Binato M. Rectal diverticulum.ABCD ArqBras CirDig.2011;24(4):33941. doi: 10.1590/S0102-67202011000400018.

6. Damron JR, Lieber A, Truman S. Rectal diverticula. Radiology. 1975;115:599-601. doi: 10.1148/15.3.599. 
7. Abrisqueta J, Frutos MD, Lujan J, Abellan I, Parrilla P. Divertículo rectal solitario. Presentacion de un caso. Cir Esp. 2013;91(2):122-31. doi: 10.1016/j.ciresp.2011.09.006.

8. Kumar R, Shun A, Arbuckle S, Gaskin K. Diverticular rectal duplication with heterotopic gastric mucosa in a child: a rare cause of rectal bleeding. J Paediatr Child Health. 2000;36:191-2. doi: 10.1046/j.1440-1754.2000.00482.x.

9. Alabiso ME, Grassi R, Fioroni C, Marano I. Iatrogenic rectal diverticulum in patients treated with transanal stapled techniques. Radiol Med. 2008;113:887-94. doi: 10.1007/ s11547-008-300-7.

10. Pellino G. Rectal diverticulum after stapled transanal procedures: surgery encouraged. Am Surg. 2013;79(12):1320.

11. Boffi F, Podzemny V. Obstructed defecation, rectocele, recurrent hemorrhoids, and rectal diverticulum following the PPH. Tech Coloproctol. 2011;15:255-6. doi: 10.1007/ s10151-011-0691-7.

12. Piercy KT, Timaran C, Akin H. Rectal diverticula. Dis Colon Rectum. 2002;45:1116-7. doi: 10.1007/s10350-004-6371-1.

13. Jung SH, Kim JH. A case of solitary rectal diverticulum presenting with a retrorectal mass. Gut Liver. 2010;4:394-7. doi: 10.5009/gnl.2010.4.3.394.
14. Kwon JH, Han KH, Chang WS, Nam KH, Han MS, Ahn $\mathrm{JH}$, et al. A Case of a Mucinous Adenocarcinoma Arising from a Rectal Diverticulum. J Korean Soc Coloproctol 2012;28(4):222-4. doi: 10.3393/jksc.2012.28.4.222.

15. Hong KD, Lee S Il, Moon HY. Rectal diverticular perforation complicating diagnostic colonoscopy: a case report and review of the literature. J Laparoendosc Adv Surg Tech A. 2011;21(8):745-8. doi: 10.1089/lap.2011.0210.

16. Martinez CAR, Priolli DG, Palma RT, Waisberg J. Divertículo do reto: relato de caso. Rev Bras Coloproct. 2003;23(4):296-30.

17. Binda GA, Cuomo R, Laghi A, Nascimbeni R, Serventi A, Bellini D, et al. Practice parameters for the treatment of colonic diverticular disease: Italian Society of Colon and Rectal Surgery (SICCR) guidelines. Tech Coloproctol. 2015;19(10):615-26. doi: 10.1007/s10151-015-1370-x.

18. Frangi A, Gomez MA, Moya P, Arroyo A, Lopez A. Rectal diverticulum resection by stapler device Tech Coloproctol. 2014;18:403-4. doi: 10.1007/s10151-012-0957-8. 